\title{
State variation in opioid treatment policies and opioid-related hospital readmissions
}

\author{
Janice Blanchard ${ }^{1,2}$, Audrey J. Weiss ${ }^{3^{*}}$, Marguerite L. Barrett ${ }^{4}$, Kimberly W. McDermott ${ }^{3}$ and Kevin C. Heslin ${ }^{5}$
}

\begin{abstract}
Background: State policy approaches designed to provide opioid treatment options have received significant attention in addressing the opioid epidemic in the United States. In particular, expanded availability of naloxone to reverse overdose, Good Samaritan laws intended to protect individuals who attempt to provide or obtain emergency services for someone experiencing an opioid overdose, and expanded coverage of medication-assisted treatment (MAT) for individuals with opioid abuse or dependence may help curtail hospital readmissions from opioids. The objective of this retrospective cohort study was to evaluate the association between the presence of state opioid treatment policies_-naloxone standing orders, Good Samaritan laws, and Medicaid medication-assisted treatment (MAT) coverage - and opioid-related hospital readmissions.
\end{abstract}

Methods: We used 2013-2015 hospital inpatient discharge data from 13 states from the Agency for Healthcare Research and Quality Healthcare Cost and Utilization Project. We examined the relationship between state opioid treatment policies and 90-day opioid-related readmissions after a stay involving an opioid diagnosis.

Results: Our sample included 383,334 opioid-related index hospitalizations. Patients treated in states with naloxone standing-order policies at the time of the index stay had higher adjusted odds of an opioid-related readmission than did those treated in states without such policies; however, this relationship was not present in states with Good Samaritan laws. Medicaid methadone coverage was associated with higher odds of readmission among all insurance groups except Medicaid. Medicaid MAT coverage generosity was associated with higher odds of readmission among the Medicaid group but lower odds of readmission among the Medicare and privately insured groups. More comprehensive Medicaid coverage of substance use disorder treatment and a greater number of opioid treatment programs were associated with lower odds of readmission.

Conclusions: Differences in index hospitalization rates suggest that states with opioid treatment policies had a higher level of need for opioid-related intervention, which also may account for higher rates of readmission. More research is needed to understand how these policies can be most effective in influencing acute care use.

Keywords: Opioid abuse, Opioid readmissions, State opioid treatment policies, Medication-assisted treatment

\section{Background}

The opioid epidemic in the United States has escalated in recent years, subsequently affecting the U.S. health care system. From 2002 to 2015, there was a 2.8-fold increase in the number of opioid-related deaths [1]. From 2005 to 2014, hospitalizations involving opioids increased by $64 \%$ [2]. Three state policy approaches designed to provide opioid treatment options have received significant attention: (1) expanded availability of

\footnotetext{
* Correspondence: audrey.weiss@us.ibm.com

${ }^{3}$ IBM Watson Health, 5425 Hollister Avenue, Suite 140, Santa Barbara, CA

93111, USA

Full list of author information is available at the end of the article
}

naloxone to reverse overdose, (2) Good Samaritan laws intended to protect individuals who attempt to provide or obtain emergency services for someone experiencing an opioid overdose, and (3) expanded coverage of medication-assisted treatment (MAT) for individuals with opioid abuse or dependence.

Naloxone can rapidly reverse the potentially life-threatening effects of opioids. It has low rates of adverse events and can be administered by laypersons [3]. Some states allow standing orders that make naloxone directly available without an individualized provider

(c) The Author(s). 2018 Open Access This article is distributed under the terms of the Creative Commons Attribution 4.0 International License (http://creativecommons.org/licenses/by/4.0/), which permits unrestricted use, distribution, and 
prescription from sites such as community agencies or pharmacies [4-6]. As of June 2016, 47 states and the District of Columbia had passed some type of law increasing naloxone availability, including laws that allow standing orders at pharmacies [5].

Good Samaritan opioid-related legislation provides individuals who call 911 for an opioid overdose with immunity from arrest, charge, or prosecution for certain drug-related violations [7]. As of January 2017, 34 states and the District of Columbia had Good Samaritan laws in place $[4,5]$.

The Food and Drug Administration (FDA) has approved three principal drugs as part of MAT to help individuals reduce the use of opioids-methadone, buprenorphine (with or without naloxone), and naltrexone (injectable and pill form). The American Society of Addiction Medicine (ASAM) guidelines recommend coverage of all three drugs $[8,9]$. Medicaid, the largest payer of substance use disorder (SUD) services, covers buprenorphine/naloxone and at least one form of naltrexone in most states; however, many states impose some restrictions such as prior authorization, dosing limits, or SUD counseling prior to prescribing [8, 9]. Many state Medicaid agencies do not cover methadone, which must be administered in an opioid treatment program (OTP) that meets federal counseling requirements $[4,9]$.

Naloxone standing orders, Good Samaritan laws, and expanded MAT coverage may affect hospitalizations related to opioid-related adverse events, overdoses, and deaths, while also encouraging individuals with opioid use disorders to seek treatment $[4,10]$. However, research evaluating the association between acute care use and these three state policies is limited, with prior studies focused only on a subset of these policies, such as coverage of methadone maintenance, or restricted to single state evaluations $[10,11]$.

The objective of this retrospective cohort study was to evaluate the relationship between these three opioid treatment policies and the risk of opioid-related readmission. We used 2013-2015 hospital discharge data from 13 states with differing treatment policies to estimate the odds of a subsequent opioid-related readmission within 90 days following discharge. We hypothesized that naloxone standing orders would be associated with lower odds of readmission because they would allow individuals with an opioid overdose to obtain treatment outside of the acute care setting, thereby reducing the need for hospitalization [4]. Conversely, we hypothesized that Good Samaritan laws may increase acute care hospital use because they would cause more individuals to alert the emergency medical system of an opioid overdose [7]. We further hypothesized that Medicaid MAT coverage would be associated with lower odds of readmission for an opioid-related diagnosis in the Medicaid population because it would increase the availability of treatment [11]. Medicaid coverage may have spillover effects because providers and facilities that treat Medicaid populations also may treat other insured populations [12] and may be more aware of MAT in states with more generous Medicaid coverage. For this reason, we hypothesized that Medicaid MAT coverage also would be associated with lower odds of readmission for patients with non-Medicaid coverage.

\section{Methods}

\section{Data source}

We used inpatient discharge data from nonfederal community hospitals in Arkansas, California, Florida, Georgia, Iowa, Maryland, Massachusetts, Nebraska, Nevada, New York, Tennessee, Vermont, and Wisconsin from the Agency for Healthcare Research and Quality (AHRQ) Healthcare Cost and Utilization Project (HCUP) [13]. We included states with encrypted patient linkage numbers to link records from the 2013, 2014, and 2015 (quarters 1 through 3) HCUP State Inpatient Databases (SID) [14] during the study period. ${ }^{1}$ We also included only those states with data indicating whether diagnoses were present on admission (POA) to exclude index stays (i.e., initial hospitalizations) involving an opioid diagnosis that may have occurred solely because of hospital-related factors, such as iatrogenic complications of opioid use.

We obtained state-level data about the status and specific implementation dates of naloxone standing orders and Good Samaritan laws from The Policy Surveillance Program: A Law Atlas Project [6, 7]. For state Medicaid MAT policies, we could determine the status of these policies for the 2013-2014 period, but specific implementation dates were unavailable. Our key sources of MAT Medicaid policy information included ASAM state reports [4, 15-27], a 2016 article by Grogan and colleagues about state Medicaid MAT benefits [9], and personal communication with the Grogan article authors. When data from these sources were incomplete, we used several supplementary sources including two state Medicaid Preferred Drug Lists [28, 29], contacts at five state Medicaid agencies, and a Kaiser Family Foundation (KFF) report on rehabilitative services [30].

We gathered information about presence of hospital detoxification and psychiatry units from the American Hospital Association [31]. For each data year, we obtained the state population capacity of facilities to treat SUDs (facilities offering care for SUDs including outpatient, residential, and inpatient hospital treatment for all payer categories), number of OTPs, and number of providers newly certified to administer buprenorphine/ naloxone from the Substance Abuse and Mental 
Health Services Administration (SAMHSA) National Survey of Substance Abuse Treatment Services [3234] and the SAMHSA Number of Drug Addiction Treatment Act (DATA)-Waived Practitioners Newly Certified Per Year tracker [35]. We also used Grogan and colleagues (2016) [9] to obtain data about state Medicaid coverage of ASAM-recommended SUD treatment levels from 2013 through 2014. Finally, we obtained state rates of opioid overdose deaths for each data year from the Kaiser Family Foundation State Health Facts database [36].

\section{Study population}

The study population comprised a retrospective longitudinal sample of patients aged 18 years and older with an opioid-related index hospitalization between April 2013 and June 2015 and no preceding opioid-related hospitalization within 90 days. ${ }^{2}$ Opioid-related stays were identified by any-listed International Classification of Diseases, Ninth Revision, Clinical Modification (ICD-9-CM) diagnosis codes that were present on admission for opioid abuse or dependence alone or in combination with other drugs (304.00-304.02, 304.70-304.72, 305.50305.52) or for poisoning by opium, methadone, heroin, opiates and related narcotics, or opiate antagonists $(965.00-965.02,965.09,970.1)$. We also included external cause of injury codes (E codes) for accidental poisoning by opium, methadone, heroin, and opiates and related narcotics (E850.0-E850.2) and adverse effects of heroin, methadone, opiates and other narcotics, and opiate antagonists (E935.0-E935.2, E940.1). We included any-listed opioid diagnoses for our index stays to capture the potential population of individuals who could be affected by naloxone, Good Samaritan, and MAT coverage policies. We excluded index hospitalizations in which the patient died or was transferred into or out of the hospital.

\section{Outcome variable: Readmissions}

The outcome variable was a readmission within a 90-day period with an opioid-related principal diagnosis or an opioid-related accidental poisoning or adverse effect diagnosis (E code). Consistent with other studies, we selected 90 days as the follow-up period because it would be sufficient time for patients discharged from the hospital to access potential outpatient rehabilitation services [37, 38]. This limited readmissions to hospitalizations that potentially would be most affected by our state policies of interest and excluded hospitalizations in which opioid-related diagnoses were only a secondary concern.

\section{Key independent variables: State policies for opioid treatment}

The key independent variables focused on the three state policies: (1) naloxone standing orders, (2) Good Samaritan laws, and (3) Medicaid MAT coverage and generosity.

The first key independent variable indicated whether a state had a naloxone standing order that allowed pharmacies to dispense naloxone without an individual provider prescription. The second indicated whether a state had a Good Samaritan law granting immunity to users from arrest, charge, or prosecution for possession of drugs or drug paraphernalia. For these first two independent variables, we classified an index stay as having a naloxone standing order or Good Samaritan law if the date of implementation was before or on the date of the index stay $[6,7]$.

The final two key independent variables represented Medicaid MAT coverage and generosity. Because the states in our sample had little variation in naltrexone and buprenorphine/naloxone coverage, we focused on two components of MAT coverage: whether a state had any coverage of methadone for Medicaid enrollees and whether a state had more or less generous coverage of buprenorphine/naloxone or naltrexone for Medicaid enrollees. Generosity of coverage of buprenorphine/naloxone or naltrexone was a composite variable based on the following four measures: (1) prior authorization requirement for buprenorphine/naloxone, (2) prior authorization requirement for injectable naltrexone, (3) buprenorphine/naloxone dosing limits (either limitation on total days coverage or maximum dosage restrictions of less than $24 \mathrm{mg} /$ day), and (4) requirement for SUD counseling prior to treatment with buprenorphine/naloxone or naltrexone. If a state lacked restrictions on at least two of these four measures, it was categorized as more (vs. less) generous. As noted above, the Medicaid methadone coverage and MAT coverage generosity measures were based on data collected from a combination of sources. Because these data sources did not provide exact dates of implementation, we counted a state as having coverage in place during the years in which our sources collected the data (2013-2014) [4, 9, 15-27].

\section{Covariates}

The analysis included covariates for patient-level factors, hospital stay and hospital characteristics, and state-level factors-all measured at the time of the index stay-that could have influenced the outcome of 90-day readmission. Patient-level factors included sociodemographic characteristics: age (continuous variable), sex, race/ethnicity (White, Black, Hispanic, other, missing), expected primary payer (Medicare, Medicaid, private insurance, uninsured/self-pay, 
other), community-level income based on the state-defined quartile for median household income of the ZIP Code of the patient's residence, and urban/ rural residency. To examine severity of illness, we identified whether the primary reason for the admission (principal diagnosis) was an opioid-related diagnosis or a non-opioid-related diagnosis, whether the patient was admitted with an opioid use disorder diagnosis (304.00-304.02, 304.70-304.72, 305.50305.52) or a poisoning/adverse effect diagnosis (965.00-965.02, 965.09, 970.1, E850.0-E850.2, E935.0E935.2, E940.1), and whether the patient had a continuous opioid use disorder $(304.01,304.71,305.51)$. We used the HCUP Clinical Classifications Software (CCS) diagnosis categories [39] to define any-listed co-occurring mental health conditions (CCS 650-652, $655-659,662,670$ ) or alcohol-related conditions (CCS 660). We used the Elixhauser Comorbidity Software [40] to create dichotomous variables indicating whether the stay involved a specific co-occurring physical (medical) condition(s); the count of the co-occurring physical condition(s) also was included.

Hospital stay characteristics included whether patients received any treatment for drug rehabilitation or detoxification during the index stay (ICD-9-CM procedure codes 94.64-94.69) and the length of the index stay. Because SUD treatment varies across hospitals, we included covariates for characteristics related to the hospital in which the index admission occurred: percentage of hospital discharges among patients with opioid-related conditions and whether the hospital had a SUD detoxification or psychiatric acute care unit.

State-level factors included the following measures of capacity for opioid treatment that were based on the year of the index stay: Medicaid coverage of all four levels of ASAM-recommended treatment services (outpatient, intensive outpatient, inpatient, intensive inpatient) during the 2013-2014 period [9]; newly certified provider capacity for buprenorphine/naloxone therapy, defined as the number of newly eligible DATA-waived practitioners approved to provide buprenorphine/naloxone treatment in a state per 100,000 population [35]; the number of OTPs per 100,000 population; and the number of SUD treatment facility beds per 100,000 population [32-34]. We included covariates for year of index stay, source of admission (emergency department vs. direct admission), and state overdose death rates to denote state-wide severity of opioid use. Because we hypothesized that the state MAT policies would have a direct effect on the Medicaid population and spillover effects on other insurance populations, we included interaction terms for Medicaid MAT coverage and generosity with each payer group.

\section{Analysis}

We first conducted bivariate analysis to examine characteristics of our sample as well as the association between our key independent (policy) variables and the outcome variable, opioid-related readmission within 90 days after discharge. Next, we conducted multivariate logistic regression analysis to estimate the association between our key independent variables and an opioid-related readmission, taking into account the patient-, hospital- and state-level factors described above.

All data were analyzed using SAS version 9.4. The HCUP databases are consistent with the definition of limited data sets under the Health Insurance Portability and Accountability Act Privacy Rule and contain no direct patient identifiers. The AHRQ Human Research Protections Program has determined that research using HCUP data has exempt status.

\section{Results \\ Bivariate analysis}

During the study period, there were 383,334 index hospitalizations involving opioid-related diagnoses across the 13 sample states. Table 1 shows characteristics of the sample of index hospital stays by each policy category. Patients treated in states with naloxone standing orders, with Good Samaritan laws, and that offered Medicaid coverage of methadone and more generous Medicaid coverage of MAT had higher rates of diagnoses of continuous opioid abuse and dependence on index stays. These patients also were more likely to be covered by Medicaid and less likely to be White compared with patients treated in states without such policies. States that offered Medicaid coverage of methadone and more generous Medicaid MAT coverage had more index stays from patients in the lower income quartiles and more stays from patients in urban areas. States with Medicaid methadone coverage had a lower capacity of newly certified providers offering office-based buprenorphine/naloxone therapy but a higher capacity of OTPs and SUD treatment facilities compared with states that did not offer such coverage. States that offered more generous MAT coverage had higher capacity of newly-certified office-based buprenorphine/naloxone providers, OTPs, and SUD facilities compared with states that offered less generous coverage.

Bivariate analysis showed that states with naloxone standing orders, Good Samaritan laws, Medicaid coverage of methadone, and more generous Medicaid coverage of MAT had a higher percentage of opioid-related readmissions within 90 days compared with states without these policies (Table 2). 
Table 1 Characteristics of opioid-related index hospitalizations by state policy category

\begin{tabular}{|c|c|c|c|c|c|c|c|c|}
\hline \multirow[t]{2}{*}{ Characteristic } & \multicolumn{2}{|c|}{$\begin{array}{l}\text { Naloxone } \\
\text { Standing Orders }\end{array}$} & \multicolumn{2}{|c|}{$\begin{array}{l}\text { Good } \\
\text { Samaritan Laws }\end{array}$} & \multicolumn{2}{|c|}{$\begin{array}{l}\text { Methadone } \\
\text { Coverage } \\
\text { by Medicaid }\end{array}$} & \multicolumn{2}{|c|}{$\begin{array}{l}\text { MAT Coverage } \\
\text { Generosity } \\
\text { by Medicaid }\end{array}$} \\
\hline & Yes & No & Yes & No & Yes & No & $\begin{array}{l}\text { More } \\
\text { Generous }\end{array}$ & $\begin{array}{l}\text { Less } \\
\text { Generous }\end{array}$ \\
\hline Number of stays & 145,262 & 238,072 & 307,369 & 75,965 & 353,335 & 29,999 & 237,221 & 146,113 \\
\hline \multicolumn{9}{|l|}{ Patient Sociodemographic Characteristics } \\
\hline Age (mean) & $47.7^{* *}$ & 46.3 & $47.0^{* *}$ & 46.0 & $46.9^{* *}$ & 45.8 & $45.0^{* *}$ & 49.7 \\
\hline Female (\%) & $48.1^{* *}$ & 49.4 & $47.7^{* *}$ & 53.7 & $48.2^{* *}$ & 57.4 & $46.5^{* *}$ & 52.8 \\
\hline \multicolumn{9}{|l|}{ Race/ethnicity (\%) } \\
\hline White & $68.6 * *$ & 70.1 & $68.6^{* *}$ & 73.2 & $68.2^{* *}$ & 85.5 & $67.9^{* *}$ & 72.2 \\
\hline Black & $12.4^{* *}$ & 14.2 & $12.8^{* *}$ & 16.5 & $14.2^{* *}$ & 5.9 & $15.3^{* *}$ & 10.7 \\
\hline Hispanic & $13.0^{* *}$ & 9.3 & $12.7^{* *}$ & 2.7 & $11.4^{* *}$ & 2.4 & $10.0^{* *}$ & 12.0 \\
\hline Other & $5.5^{* *}$ & 4.1 & $5.3^{* *}$ & 1.9 & $4.9^{* *}$ & 0.6 & $5.3^{* *}$ & 3.5 \\
\hline Missing & $0.5^{* *}$ & 2.3 & $0.6^{* *}$ & 5.7 & $1.3^{* *}$ & 5.5 & 1.6 & 1.6 \\
\hline \multicolumn{9}{|l|}{ Expected primary payer (\%) } \\
\hline Medicare & $31.5^{* *}$ & 30.1 & $30.5^{* *}$ & 31.4 & $30.2^{* *}$ & 36.1 & $27.0^{* *}$ & 36.5 \\
\hline Medicaid & $42.4^{* *}$ & 34.7 & $38.9^{* *}$ & 32.5 & $38.6^{* *}$ & 26.1 & $42.3^{* *}$ & 30.0 \\
\hline Private insurance & $18.0^{* *}$ & 17.5 & $17.7^{*}$ & 17.4 & $17.5^{* *}$ & 17.5 & $16.6^{* *}$ & 19.3 \\
\hline Uninsured/self-pay & $5.1^{* *}$ & 13.2 & $8.8^{* *}$ & 15.5 & $9.7^{* *}$ & 14.7 & $10.9^{* *}$ & 8.8 \\
\hline Other & $3.0^{* *}$ & 4.4 & $4.1^{* *}$ & 2.8 & $4.0^{* *}$ & 2.0 & $3.1^{* *}$ & 5.1 \\
\hline \multicolumn{9}{|l|}{ Median community-level income (\%) } \\
\hline Quartile 1 (lowest) & $31.7^{* *}$ & 34.9 & $32.4^{* *}$ & 38.9 & $34.0^{* *}$ & 29.9 & $35.9^{* *}$ & 30.1 \\
\hline Quartile 2 & $24.6^{* *}$ & 23.8 & $23.9^{* *}$ & 25.0 & $23.7^{* *}$ & 28.8 & $22.6^{* *}$ & 26.5 \\
\hline Quartile 3 & $21.1^{*}$ & 20.8 & $21.1^{* *}$ & 20.2 & $20.6^{* *}$ & 24.2 & $19.9^{* *}$ & 22.5 \\
\hline Quartile 4 (highest) & $17.6^{* *}$ & 16.4 & $17.5^{* *}$ & 14.3 & $16.9^{* *}$ & 15.7 & $16.5^{* *}$ & 17.4 \\
\hline Missing & $5.0^{* *}$ & 4.1 & $5.2^{* *}$ & 1.6 & $4.7^{* *}$ & 1.4 & $5.1^{* *}$ & 3.4 \\
\hline \multicolumn{9}{|l|}{ Patient location (\%) } \\
\hline Urban & $89.7^{* *}$ & 90.7 & $92.4^{* *}$ & 82.0 & $92.0^{* *}$ & 70.9 & $93.1^{* *}$ & 85.8 \\
\hline Rural & $8.9^{* *}$ & 8.2 & $6.2^{* *}$ & 17.7 & $6.7^{* *}$ & 28.9 & $5.9^{* *}$ & 12.6 \\
\hline \multicolumn{9}{|l|}{ Patient Clinical Factors } \\
\hline \multicolumn{9}{|l|}{ Opioid-related diagnosis (\%) } \\
\hline Principal & $13.3^{* *}$ & 13.8 & $13.7^{*}$ & 13.4 & $13.3^{* *}$ & 17.0 & $14.4^{* *}$ & 12.2 \\
\hline Secondary only & $86.7^{* *}$ & 86.2 & $86.3^{*}$ & 86.6 & $86.7^{* *}$ & 83.0 & $85.6^{* *}$ & 87.8 \\
\hline Any opioid abuse or dependence (\%) & 83.0 & 82.9 & $83.6^{* *}$ & 80.0 & $83.4^{* *}$ & 77.4 & $85.4^{* *}$ & 78.8 \\
\hline Continuous opioid abuse or dependence (\%) & $29.9^{*}$ & 29.6 & $30.8^{* *}$ & 25.2 & $30.4^{* *}$ & 21.4 & $32.6^{* *}$ & 24.9 \\
\hline Mental health co-occurring diagnosis (\%) & $54.1^{* *}$ & 55.7 & $54.2^{* *}$ & 58.9 & $54.6^{* *}$ & 61.3 & $55.7^{* *}$ & 54.2 \\
\hline Alcohol abuse co-occurring diagnosis (\%) & 19.5 & 19.5 & $19.7^{* *}$ & 18.6 & $19.9^{* *}$ & 14.5 & $21.3^{* *}$ & 16.5 \\
\hline Number of co-occurring physical conditions (mean) ${ }^{b}$ & $2.1^{* *}$ & 2.0 & $2.0^{* *}$ & 2.1 & $2.0^{* *}$ & 1.9 & $1.8^{* *}$ & 2.3 \\
\hline \multicolumn{9}{|l|}{ Initial Hospital Stay Characteristics } \\
\hline Hospital stay began in ED (mean \%) & $75.0^{* *}$ & 76.7 & $77.4^{* *}$ & 70.9 & $77.2 * *$ & 63.5 & $76.4^{* *}$ & 75.5 \\
\hline Length of index stay (mean days) & $5.3^{* *}$ & 5.1 & $5.3^{* *}$ & 4.6 & $5.2^{* *}$ & 4.5 & $5.2^{* *}$ & 5.1 \\
\hline Receipt of rehabilitation during index stay (\%) & $16.9^{* *}$ & 16.1 & $16.9^{* *}$ & 14.7 & 16.5 & 16.3 & $21.2^{* *}$ & 8.8 \\
\hline \multicolumn{9}{|l|}{ Hospital Characteristics ${ }^{c}$} \\
\hline Percentage of annual opioid-related discharges in index year (mean) & 3.9 & 3.9 & 3.9 & 3.9 & $3.9^{* *}$ & 3.2 & $4.7^{* *}$ & 2.5 \\
\hline
\end{tabular}


Table 1 Characteristics of opioid-related index hospitalizations by state policy category (Continued)

\begin{tabular}{|c|c|c|c|c|c|c|c|c|}
\hline \multirow[t]{2}{*}{ Characteristic } & \multicolumn{2}{|c|}{$\begin{array}{l}\text { Naloxone } \\
\text { Standing Orders }\end{array}$} & \multicolumn{2}{|c|}{$\begin{array}{l}\text { Good } \\
\text { Samaritan Laws }\end{array}$} & \multicolumn{2}{|c|}{$\begin{array}{l}\text { Methadone } \\
\text { Coverage } \\
\text { by Medicaid } \\
\end{array}$} & \multicolumn{2}{|c|}{$\begin{array}{l}\text { MAT Coverage } \\
\text { Generosity } \\
\text { by Medicaid }\end{array}$} \\
\hline & Yes & No & Yes & No & Yes & No & $\begin{array}{l}\text { More } \\
\text { Generous }\end{array}$ & $\begin{array}{l}\text { Less } \\
\text { Generous }\end{array}$ \\
\hline \multicolumn{9}{|l|}{ Alcohol/substance use disorder (SUD) detoxification unit (\%) } \\
\hline No & $56.8^{* *}$ & 59.3 & $57.5^{* *}$ & 61.8 & $58.0^{* *}$ & 62.1 & $53.0^{* *}$ & 67.0 \\
\hline Yes & $26.7^{* *}$ & 24.3 & $25.9 * *$ & 22.3 & $25.7^{* *}$ & 19.4 & $32.8^{* *}$ & 12.8 \\
\hline Not reported & 16.6 & 16.4 & $16.6^{* *}$ & 15.9 & $16.3^{* *}$ & 18.5 & $14.2^{* *}$ & 20.1 \\
\hline \multicolumn{9}{|l|}{ Psychiatric acute care unit (\%) } \\
\hline No & $35.9^{* *}$ & 30.9 & $32.9^{*}$ & $32.5^{*}$ & $32.7^{* *}$ & 34.7 & $25.7^{* *}$ & 44.4 \\
\hline Yes & $47.5^{* *}$ & 52.8 & $50.6^{* *}$ & 51.6 & $51.1^{* *}$ & 46.8 & $60.1^{* *}$ & 35.7 \\
\hline Not reported & $16.6^{*}$ & 16.3 & $16.5^{* *}$ & 15.9 & $16.2^{* *}$ & 18.5 & $14.2^{* *}$ & 19.9 \\
\hline \multicolumn{9}{|l|}{ State Factors } \\
\hline Medicaid coverage of ASAM recommended treatment services (mean) & $87.1^{* *}$ & 58.7 & $72.0^{* *}$ & 59.1 & $69.2^{* *}$ & 72.6 & $65.0^{* *}$ & 76.6 \\
\hline $\begin{array}{l}\text { Newly certified provider capacity for buprenorphine/naloxone per } \\
100,000 \text { state population during year of index stay (mean) }\end{array}$ & $86.2^{* *}$ & 76.4 & $79.9^{* *}$ & 80.9 & $78.8^{* *}$ & 96.1 & $95.6^{* *}$ & 55.0 \\
\hline $\begin{array}{l}\text { Number of opioid treatment programs per 100,000 state population } \\
\text { during year of index stay (mean) }\end{array}$ & $0.6^{*}$ & 0.6 & $0.6^{* *}$ & 0.5 & $0.6^{* *}$ & 0.1 & $0.7^{* *}$ & 0.3 \\
\hline $\begin{array}{l}\text { SUD treatment capacity per 100,000 state population during year of } \\
\text { index stay (mean) }\end{array}$ & $43.4^{* *}$ & 42.2 & $45.2^{* *}$ & 32.4 & $43.7^{* *}$ & 30.5 & $48.6^{* *}$ & 33.0 \\
\hline Opioid overdose death rate during year of index stay (mean) & $9.1^{* *}$ & 9.3 & $8.7^{* *}$ & 11.4 & $9.1^{* *}$ & 11.0 & $10.7^{* *}$ & 6.9 \\
\hline
\end{tabular}

ASAM American Society of Addiction Medicine, MAT medication-assisted treatment, OTP opioid treatment program, SUD substance use disorder ${ }^{*} p<0.05 ;{ }^{* *} p<0.001$

${ }^{a}$ This is a composite variable of preauthorization requirements for buprenorphine/naloxone, preauthorization for naltrexone, dosing limits for buprenorphine/ naltrexone, and SUD counseling requirements

${ }^{\mathrm{b}}$ Co-occurring conditions were formulated from 25 Elixhauser comorbidities (excluding mental health and SUD variables, which are reported as separate variables)

'Hospital characteristics describe the hospital at which the index visit occurred

Table 2 Bivariate results: unadjusted association of 90-day readmission and state policy

\begin{tabular}{|c|c|c|}
\hline State Policy & $\begin{array}{l}\% \text { Readmitted } \\
\text { Within } 90 \text { Days }\end{array}$ & $95 \% \mathrm{Cl}$ \\
\hline \multicolumn{3}{|c|}{ Naloxone standing orders } \\
\hline Yes & $3.2^{*}$ & $3.1-3.2$ \\
\hline No & 2.8 & $2.8-2.9$ \\
\hline \multicolumn{3}{|c|}{ Good Samaritan laws } \\
\hline Yes & $3.2^{*}$ & $3.2-3.3$ \\
\hline No & 1.7 & $1.6-1.8$ \\
\hline \multicolumn{3}{|c|}{ Methadone coverage by Medicaid } \\
\hline Yes & $3.0^{*}$ & $3.0-3.1$ \\
\hline No & 1.8 & $1.6-1.9$ \\
\hline \multicolumn{3}{|c|}{ MAT coverage generosity by Medicaid ${ }^{a}$} \\
\hline More generous & $3.5^{*}$ & $3.5-3.6$ \\
\hline Less generous & 2.0 & $1.9-2.1$ \\
\hline
\end{tabular}

$\mathrm{Cl}$ confidence interval, MAT, medication-assisted treatment

${ }^{*} p<0.001$

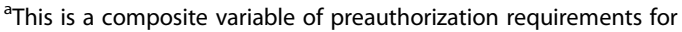
buprenorphine/naloxone, preauthorization for naltrexone, dosing limits for buprenorphine/naltrexone, and substance use disorder

counseling requirements

\section{Multivariate analysis}

Table 3 shows the results of our multivariate analysis. Patients in states with naloxone standing orders had higher odds of an opioid-related readmission ( $\mathrm{OR}=1.14$, $95 \% \mathrm{CI}=1.07-1.20)$ compared with patients in states without naloxone standing orders. There was no significant relationship between Good Samaritan laws and opioid-related readmission.

By insurance group, compared with states without Medicaid methadone coverage, the odds of readmission in states with Medicaid methadone coverage were higher among Medicare patients $(\mathrm{OR}=1.40,95 \% \mathrm{CI}=1.15-$ $1.72)$, privately insured patients $(\mathrm{OR}=1.87,95 \% \mathrm{CI}=$ 1.44-2.41), and uninsured patients $(\mathrm{OR}=1.57,95 \% \mathrm{CI}=$ 1.06-2.33). Medicaid methadone coverage was not associated with readmission among Medicaid patients.

The odds of readmission in states with more generous Medicaid MAT coverage were lower among Medicare and privately insured patients $(\mathrm{OR}=0.80,95 \% \mathrm{CI}=0.70-0.91$; $\mathrm{OR}=0.74,95 \% \mathrm{CI}=0.63-0.85$, respectively) but higher among Medicaid patients $(\mathrm{OR}=1.28,95 \% \mathrm{CI}=1.11-1.48)$ compared with these same groups of patients in states with less generous Medicaid MAT coverage. Medicaid MAT coverage generosity was not associated with readmissions among uninsured patients. 
Table 3 Multivariate results: adjusted association of 90-day readmission and state policies ${ }^{a}$

\begin{tabular}{|c|c|c|}
\hline \multirow[t]{2}{*}{ Variable } & \multicolumn{2}{|c|}{ 90-Day Readmission } \\
\hline & $\mathrm{OR}$ & $95 \% \mathrm{Cl}$ \\
\hline \multicolumn{3}{|l|}{ Key Independent Variables } \\
\hline Naloxone standing orders & $1.14^{* * *}$ & $1.07-1.20$ \\
\hline Good Samaritan laws & 1.07 & $0.97-1.19$ \\
\hline \multicolumn{3}{|l|}{$\begin{array}{l}\text { Methadone coverage by Medicaid, by payer } \\
\text { (reference: without methadone coverage) }^{b}\end{array}$} \\
\hline Medicaid & 1.23 & $0.97-1.56$ \\
\hline Medicare & $1.40^{* *}$ & $1.15-1.72$ \\
\hline Private insurance & $1.87^{* * *}$ & $1.44-2.41$ \\
\hline Uninsured & $1.57^{*}$ & $1.06-2.33$ \\
\hline Other insurance/missing & 0.99 & $0.56-1.72$ \\
\hline \multicolumn{3}{|l|}{ 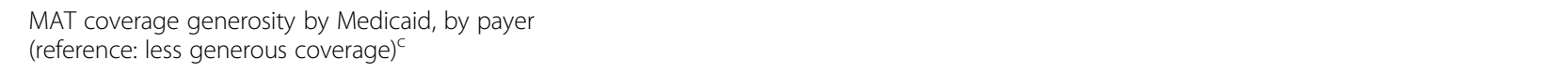 } \\
\hline Medicaid & $1.28^{* *}$ & $1.11-1.48$ \\
\hline Medicare & $0.80^{* *}$ & $0.70-0.91$ \\
\hline Private insurance & $0.74^{* * *}$ & $0.63-0.85$ \\
\hline Uninsured & 1.20 & $0.95-1.51$ \\
\hline Other insurance/missing & 1.14 & $0.86-1.50$ \\
\hline \multicolumn{3}{|l|}{ Covariates } \\
\hline \multicolumn{3}{|l|}{ Patient Sociodemographic Characteristics } \\
\hline Age & $0.99^{* * *}$ & $0.99-0.99$ \\
\hline Female & $0.86^{* * *}$ & $0.83-0.90$ \\
\hline \multicolumn{3}{|l|}{ Race/ethnicity (reference: White) } \\
\hline Black & 0.97 & $0.91-1.03$ \\
\hline Hispanic & 1.05 & $0.98-1.11$ \\
\hline Other & 0.97 & $0.89-1.05$ \\
\hline Missing & $0.78^{*}$ & $0.63-0.97$ \\
\hline \multicolumn{3}{|l|}{$\begin{array}{l}\text { Median community-level income } \\
\text { (reference: Quartile 4, highest) }\end{array}$} \\
\hline Quartile 1 (lowest) & $0.87^{* * *}$ & $0.82-0.92$ \\
\hline Quartile 2 & $0.84^{* * *}$ & $0.79-0.89$ \\
\hline Quartile 3 & $0.87^{* * *}$ & $0.82-0.92$ \\
\hline Missing & 0.95 & $0.86-1.05$ \\
\hline Patient location: rural (reference: urban) & $0.84^{* * *}$ & $0.78-0.92$ \\
\hline \multicolumn{3}{|l|}{ Patient Clinical Factors } \\
\hline $\begin{array}{l}\text { Opioid-related diagnosis: principal } \\
\text { (reference: secondary diagnosis only) }\end{array}$ & $1.88^{* * *}$ & $1.79-1.98$ \\
\hline Any opioid abuse or dependence & $0.83^{* * *}$ & $0.76-0.90$ \\
\hline Continuous opioid abuse or dependence & $1.16^{* * *}$ & $1.10-1.21$ \\
\hline Mental health co-occurring diagnosis & $1.09^{* *}$ & $1.03-1.15$ \\
\hline Alcohol abuse co-occurring diagnosis & 1.01 & $0.96-1.06$ \\
\hline Number of co-occurring physical conditions ${ }^{d}$ & $1.29^{* *}$ & $1.08-1.53$ \\
\hline \multicolumn{3}{|l|}{ Initial Hospital Stay Characteristics } \\
\hline Hospital stay began in emergency department & $1.16^{* * *}$ & $1.11-1.22$ \\
\hline Length of index stay & $0.98^{* * *}$ & $0.98-0.99$ \\
\hline Receipt of rehabilitation during index stay & $1.96^{* * *}$ & $1.84-2.09$ \\
\hline
\end{tabular}


Table 3 Multivariate results: adjusted association of 90-day readmission and state policies ${ }^{\mathrm{a}}$ (Continued)

\begin{tabular}{|c|c|c|}
\hline \multirow[t]{2}{*}{ Variable } & \multicolumn{2}{|c|}{ 90-Day Readmission } \\
\hline & $\mathrm{OR}$ & $95 \% \mathrm{Cl}$ \\
\hline \multicolumn{3}{|l|}{ Hospital Characteristics } \\
\hline Percentage of annual opioid-related discharges in index year & $1.02^{* * *}$ & $1.02-1.03$ \\
\hline \multicolumn{3}{|l|}{ Alcohol/ SUD detoxification unit (reference: no unit) } \\
\hline Yes & $1.27^{* * *}$ & $1.20-1.34$ \\
\hline Not reported & 0.90 & $0.33-2.43$ \\
\hline \multicolumn{3}{|l|}{ Psychiatric acute care unit (reference: no unit) } \\
\hline Yes & 1.01 & $0.96-1.06$ \\
\hline Not reported & 1.23 & $0.45-3.33$ \\
\hline \multicolumn{3}{|l|}{ State Factors } \\
\hline Medicaid coverage of all four ASAM recommended services & $0.74^{* * *}$ & $0.66-0.82$ \\
\hline $\begin{array}{l}\text { Newly certified provider capacity for buprenorphine/naloxone per } 1 \\
00,000 \text { state population during year of index stay }\end{array}$ & $1.00^{*}$ & $1.00-1.00$ \\
\hline $\begin{array}{l}\text { Number of opioid treatment programs per 100,000 state population } \\
\text { during year of index stay }\end{array}$ & $0.35^{* * *}$ & $0.28-0.44$ \\
\hline $\begin{array}{l}\text { SUD treatment capacity per } 100,000 \text { state population during year of } \\
\text { index stay }\end{array}$ & $1.04^{* * *}$ & $1.03-1.04$ \\
\hline Opioid overdose death rate during year of index stay & $1.02^{*}$ & $1.01-1.04$ \\
\hline \multicolumn{3}{|c|}{$\begin{array}{l}\text { ASAM American Society of Addiction Medicine, } C I \text { confidence interval, MAT medication-assisted treatment, OR odds ratio, SL } \\
{ }^{*} p<0.05 ;{ }^{* *} p<0.01 ; * * * p<0.001 \\
\text { aThe model also controlled for co-occurring physical conditions and year of discharge, which are not listed in the table } \\
\text { b Odds ratios were calculated by using an interaction term for Medicaid methadone coverage and each of the payer groups }\end{array}$} \\
\hline
\end{tabular}

Among the covariates in our model, patients treated in states where Medicaid covered all four ASAM-recommended treatment levels and states with more OTPs had lower odds of an opioid-related readmission $(\mathrm{OR}=0.74,95 \% \mathrm{CI}=0.66-0.82$; $\mathrm{OR}=0.35$, $95 \% \mathrm{CI}=0.28-0.44$, respectively) than did patients in states without these capacities. By contrast, patients in states with more substance use facility beds (which included residential and inpatient beds for all payers) and a higher opioid death rate had higher odds of readmission $(\mathrm{OR}=1.04,95 \% \mathrm{CI}=1.03-1.04 ; \mathrm{OR}=1.02$, 95\% CI $=1.01-1.04$, respectively).

Other factors associated with higher odds of an opioid-related readmission included the following at index hospitalization: a continuous opioid-related diagnosis, an opioid principal diagnosis, more physical codiagnoses, a mental health codiagnosis, and receipt of inpatient detoxification or rehabilitation treatment. In addition, patients with index stays in hospitals with more opioid-related discharges or with dedicated SUD treatment units had higher odds of readmission. By contrast, patients who were female, from the lowest income quartile or rural areas, with an opioid use disorder diagnosis (vs. a poisoning or adverse event diagnosis), or with a longer index stay had lower odds of readmission.

\section{Discussion}

In this study, patients with an opioid-related hospitalization in states with naloxone standing orders, more generous Medicaid methadone coverage (for the privately insured, Medicare, and uninsured groups), and more generous Medicaid MAT coverage (for the Medicaid group) had higher odds of an opioid-related readmission within 90 days. It is likely that policies that address opioid use disorders, such as the ones we explored, were implemented in states that had high need for opioid-related intervention. For example, states with more generous Medicaid MAT coverage had higher opioid death rates. Patients in states with these policies were more likely to be admitted for continuous opioid use and more likely to be covered by Medicaid than patients from states without these policies. Another possible explanation for our findings is that states that implemented these policies had more aggressive lobbying efforts to promote passage of such laws coupled with educational campaigns that resulted in an increase in 
patient awareness about the importance of acute treatment for opioids [41].

Contrary to our hypothesis, patients in states with naloxone standing orders were more likely to be readmitted for opioid use than were patients in states without these policies. Prior research has shown that naloxone may reduce overdose mortality [42]. For example, a Massachusetts study showed that communities that distributed nasal naloxone had a reduction in overdose deaths compared with those that did not [10]. One explanation for our study's findings is that by preventing deaths outside of the hospital, naloxone access may allow individuals to survive the initial opioid overdose and get to the hospital to seek care.

We did not find any relationship between Good Samaritan laws and opioid-related readmissions. We may not have had sufficient time to see the effects of these laws, many of which were implemented recently [7]. Despite the increasing adoption of Good Samaritan laws, many individuals who are at the greatest risk of opioid overdose are unaware of their existence or scope. A 2015 survey in Rhode Island of young adults who reported nonmedical use of prescription opioids showed that fewer than half knew about the state's Good Samaritan law, enacted in 2012 [43]. To be most effective, it is likely that these laws need to be accompanied by educational campaigns alerting potential beneficiaries of their existence.

This analysis showed associations of varying magnitude and significance, depending on insurance coverage, between opioid-related readmission and Medicaid coverage of methadone and Medicaid MAT coverage generosity. Methadone coverage did not have a statistically significant association with readmission in the Medicaid population. However, in all other payer populations, individuals treated in states with Medicaid methadone coverage had higher odds of readmission. In prior studies among the Medicaid population, methadone use has been associated with an increased likelihood of acute care use for overdoses from opioids [44-46]. When patients present to OTPs for methadone, they have access to providers who may identify the need for acute care for opioid-related diagnoses that may warrant medical intervention. Medicaid coverage of methadone may help provide funding to OTPs that allow patients, regardless of insurance status, to obtain such access to care $[4,12]$. In 2003, after Oregon temporarily discontinued Medicaid coverage of methadone, providers reported decreases in staff support and services related to the loss of this funding [12].

Medicaid MAT coverage generosity was associated with lower odds of an opioid-related readmission among individuals covered by Medicare or private insurance but higher odds of readmission for those covered by
Medicaid. The reason is not entirely clear. The Medicaid population historically has had high rates of readmission, particularly for SUDs [38]. Our finding may reflect lower access to resources needed for outpatient rehabilitation services and coordinated care after an acute care episode among individuals covered by Medicaid [38, 47]. Individuals covered by private insurance and Medicare in states with generous Medicaid MAT coverage may be seeing benefits to therapy not otherwise realized among individuals covered by Medicaid, suggesting some spillover effects to privately insured and Medicare beneficiaries (e.g., because of greater provider awareness of MAT), perhaps combined with better access to other outpatient support services.

The findings also demonstrated that outpatient treatment capacity, along with coverage, is an important factor associated with readmission, particularly among the Medicaid population. Many states face barriers to distribution of MAT that extend beyond coverage, including lack of available providers, which tends to be most severe for publicly funded facilities [48]. In this analysis, individuals in states with more OTP facilities that distribute methadone, and often other forms of MAT, had lower odds of readmission. In addition, individuals in states where Medicaid covered all four ASAM levels of SUD treatment had lower odds of readmission. Even if a state offers Medicaid coverage of MAT, individuals in states with low availability of the types of providers needed to deliver MAT likely would not benefit from these coverage policies. After Massachusetts expanded coverage of SUD services, use of the services remained essentially flat-possibly because of a lack of expansion of infrastructure or sufficient engagement of clients [49].

Our study had some limitations. We did not have information in our sample on individual patient treatment or on whether individuals received treatment outside of the acute care medical or surgical hospital setting. We also did not have information on events that occurred outside of the acute care setting, such as post-discharge deaths, that may have affected our outcomes. Although we had the exact dates of implementation for naloxone standing orders and Good Samaritan laws, we did not have the exact dates of implementation for Medicaid methadone or MAT coverage policies. For the latter, we relied on general implementation information for the range of years during our study's data collection period. Another limitation is that the association between our outcome and our policy variables may have been related to unmeasured variables; in addition, there may have been unmeasured endogenous terms that may have similarly impacted both the outcome and predictor variables. In addition, the available policy information did not distinguish between Medicaid fee-for-service and managed care for most states in our sample. Medicaid managed 
care plans may have separate formularies from fee-for-service formularies that may involve medication restrictions and prior authorization and utilization review requirements. Our sources indicated, however, that states in our sample had similar SUD coverage requirements under both types of plans. Finally, since our analysis was limited to 13 states, our results may not be generalizable to other regions or the nation as a whole.

\section{Conclusions}

Policies to address opioid use disorders such as naloxone standing orders, Good Samaritan laws, and MAT coverage are still in their nascent stages but have the potential to affect acute care utilization, including readmissions. The implementation of such policies in the states included in this study may have been motivated by higher rates of continuous opioid abuse and dependence in the population. Although some policies were associated with increased rates of readmissions, others were associated with a decrease or no change in readmission rates. We propose some explanations for our findings; however, more research is needed to understand how these policies can be most effective in influencing acute care utilization, including educating the public about the existence of these benefits and ensuring that states have adequate capacity to optimize their effectiveness.

\section{Endnotes}

${ }^{1}$ We did not include data for quarter 4 of 2015 because of the transition during this time from the ICD-9-CM to the ICD-10-CM/PCS coding system, which previous research suggests may have issues with compatibility [50].

${ }^{2}$ We used hospital discharges in January through March 2013 only for assessment of the 90-day pre-index period and discharges from July through September 2015 only for assessment of the 90-day post-index readmission period.

\footnotetext{
Abbreviations

AHRQ: Agency for Healthcare Research and Quality; ASAM: American Society of Addiction Medicine; CCS: Clinical Classifications Software; DATA: Drug Addiction Treatment Act; FDA: The Food and Drug Administration; HCUP: Healthcare Cost and Utilization Project; ICD-9-CM: International Classification of Diseases, Ninth Revision, Clinical Modification:

MAT: Medication-assisted treatment; OTP: Opioid treatment program; POA: Present on admission; SID: State Inpatient Databases; SUD: Substance use disorder
}

\section{Acknowledgements}

The authors gratefully acknowledge Minya Sheng, MS (IBM Watson Health) for assistance in programming and data management; Eli Cutler, PhD (IBM Watson Health) and Frank Yoon, PhD (IBM Watson Health) for providing statistical guidance; Rosanna Coffey, PhD (IBM Watson Health) for providing topical expertise and insights; and Linda Lee, PhD (IBM Watson Health) and Paige Jackson, MS (IBM Watson Health) for providing editorial review of the manuscript. We would like to thank Colleen Grogan, PhD and other members of the National Drug Abuse Treatment System Survey (NDATSS) research team for contributing to our analysis by sharing Medicaid MAT policy data for the 2013-2014 period.

We also wish to acknowledge the 13 Healthcare Cost and Utilization Project (HCUP) Partner organizations that contributed to the 2013-2015 HCUP State Inpatient Databases (SID) used in this study: Arkansas Department of Health, California Office of Statewide Health Planning and Development, Florida Agency for Health Care Administration, Georgia Hospital Association, lowa Hospital Association, Maryland Health Services Cost Review Commission, Massachusetts Center for Health Information and Analysis, Nebraska Hospital Association, Nevada Department of Health and Human Services, New York State Department of Health, Tennessee Hospital Association, Vermont Association of Hospitals and Health Systems, and Wisconsin Department of Health Services.

\section{Funding}

This research was supported by the Agency for Healthcare Research and Quality (AHRQ), Center for Delivery, Organization, and Markets, Healthcare Cost and Utilization Project (HCUP). One of the study authors is an employee of $A H R Q$ (K.C.H), and he contributed to all aspects of the study as a collaborator with the authors who are not employees of AHRQ (J.B., A.J.W., M.L.B., K.W.M.). The views expressed in this article are those of the authors and do not necessarily reflect those of AHRQ or the U.S. Department of Health and Human Services.

\section{Availability of data and materials}

The data that support the findings of this study are available from the Agency for Healthcare Quality and Research but restrictions apply to the availability of these data, which were used under license for the current study, and so are not publicly available.

\section{Authors' contributions}

All authors, J.B., A.J.W., M.L.B., K.W.M. and K.C.H., made substantial contributions to the design, analysis, and interpretation of results of this study and completion of the manuscript. All authors read and approved the final manuscript.

Ethics approval and consent to participate

The HCUP databases are consistent with the definition of limited data sets under the Health Insurance Portability and Accountability Act Privacy Rule and contain no direct patient identifiers. The AHRQ Human Research Protections Program has determined that research using HCUP data has exempt status.

\section{Consent for publication}

Not applicable.

\section{Competing interests}

The authors declare that they have no competing interests.

\section{Publisher's Note}

Springer Nature remains neutral with regard to jurisdictional claims in published maps and institutional affiliations.

\section{Author details}

${ }^{1}$ RAND Corporation, 1200 Hayes Street, Arlington, VA 22202, USA. ${ }^{2}$ George Washington University, 2120 L Street NW, Suite 450, Washington, DC 20037, USA. ${ }^{3}$ IBM Watson Health, 5425 Hollister Avenue, Suite 140, Santa Barbara, CA 93111, USA. ${ }^{4}$ ML Barrett, Inc., 13943 Boquita Drive, Del Mar, CA 92014, USA. ${ }^{5}$ Agency for Healthcare Research and Quality, 5600 Fishers Lane, Rockville, MD 20857, USA.

Received: 4 June 2018 Accepted: 12 November 2018 Published online: 17 December 2018

\section{References}

1. National Institute on Drug Abuse, 2017. Overdose death rates. https:// wwwdrugabusegov/related-topics/trends-statistics/overdose-death-rates Accessed 03 April 17.

2. Weiss, A.J., Elixhauser, A. Barrett, M.L., Steiner, C.A., Bailey, M.K., O'Malley, L., 2016. Opioid-Related Inpatient Stays and Emergency Department Visits by State, 2009-2014. HCUP statistical brief \#219. Agency for 
Healthcare Research and Quality. http://www.hcup-us.ahrq.gov/reports/ statbriefs/sb219-Opioid-Hospital-Stays-ED-Visits-by-State.pdf. Accessed 27 April 2018.

3. Lim JK, Bratberg JP, Davis CS, Green TC, Walley AY. Prescribe to prevent: overdose prevention and naloxone rescue kits for prescribers and pharmacists. J Addict Med. 2016;10:300-8. https://doi.org/10.1097/ADM. 0000000000000223.

4. American Society of Addiction Medicine, 2013. Advancing Access to Addiction Medications: Implications for Opioid Addiction Treatment. http:// www.asam.org/docs/default-source/advocacy/aaam_implications-for-opioidaddiction-treatment_final. Accessed 24 April 2018.

5. Network of Public Health Law, 2017. Using Law to Support Pharmacy Naloxone Distribution. https://www.networkforphl.org/_asset/qdkn97/ Pharmacy-Naloxone-Distributions.pdf. Accessed 24 April 2018.

6. Policy Surveillance Program: A Law Atlas Project, n.d.b. Naloxone Overdose Prevention Laws. http://lawatlas.org/page/naloxone-overdose-preventionlaws. Accessed 25 May 2017.

7. Policy Surveillance Program: A Law Atlas Project, n.d.a. Good Samaritan Overdose Prevention Laws. http://lawatlas.org/page/good-samaritanoverdose-prevention-laws. Accessed 25 May 2017.

8. American Society of Addiction Medicine, 2015. The National Practice Guideline for the Use of Medications in the Treatment of Addiction Involving Opioid Use. http://www.asam.org/docs/default-source/practicesupport/guidelines-and-consensus-docs/asam-national-practice-guidelinesupplement.pdf. (Accessed 08 April 2018).

9. Grogan CM, Andrews C, Abraham A, Humphreys K, Pollack HA, Smith BT, Friedmann PD. Survey highlights differences in Medicaid coverage for substance use treatment and opioid use disorder medications. Health Aff. 2016;35:2289-96. https://doi.org/10.1377/hlthaff.2016.0623.

10. Walley AY, Xuan Z, Hackman HH, Quinn E, Doe-Simkins M, SorensenAlawad A, Ruiz S, Ozonoff A. Opioid overdose rates and implementation of overdose education and nasal naloxone distribution in Massachusetts: interrupted time series analysis. BMJ. 2013;346:f174. https://doi.org/10.1136/bmj.f174.

11. Saloner B, Stoller KB, Barry CL. Medicaid coverage for methadone maintenance and use of opioid agonist therapy in specialty addiction treatment. Psych Serv. 2016;67:676-9. https://doi.org/10.1176/appi.ps.201500228.

12. Deck DD, Wiitala WL, Laws KE. Medicaid coverage and access to publicly funded opiate treatment. J Behav Health Serv Res. 2006;33:321-34. https:// doi.org/10.1007/s11414-006-9018-2.

13. Agency for Healthcare Research and Quality, 2016a. Overview of HCUP. https://www.hcup-us.ahrq.gov/overview.jsp. Accessed 24 April 2018.

14. Agency for Healthcare Research and Quality, 2016b. Overview of the State Inpatient Databases (SID). http://www.hcup-us.ahrq.gov/sidoverview.jsp. Accessed 24 April 2018.

15. American Society of Addiction Medicine, 2014a. Medicaid Coverage of Medications for the Treatment of Opioid Use Disorder: Arkansas. http:// www.asam.org/docs/default-source/advocacy/State-medicaid-reports/Statemedicaid-reports ar.pdf. Accessed 01 March 2017.

16. American Society of Addiction Medicine, 2014b. Medicaid Coverage of Medications for the Treatment of Opioid Use Disorder: California. https:// www.asam.org/docs/default-source/advocacy/State-medicaid-reports/Statemedicaid-reports_ca.pdf. Accessed 01 March 2017.

17. American Society of Addiction Medicine, 2014c. Medicaid Coverage of Medications for the Treatment of Opioid Use Disorder: Florida. http://www. asam.org/docs/default-source/advocacy/State-medicaid-reports/Statemedicaid-reports_fl.pdf. Accessed 01 March 2017.

18. American Society of Addiction Medicine, 2014d. Medicaid Coverage of Medications for the Treatment of Opioid Use Disorder: Georgia. http://www. asam.org/docs/default-source/advocacy/State-medicaid-reports/Statemedicaid-reports_ga.pdf. Accessed 01 March 2017.

19. American Society of Addiction Medicine, 2014e. Medicaid Coverage of Medications for the Treatment of Opioid Use Disorder: lowa. http://www. asam.org/docs/default-source/advocacy/State-medicaid-reports/Statemedicaid-reports_ia.pdf. Accessed 01 March 2017.

20. American Society of Addiction Medicine, 2014f. Medicaid Coverage of Medications for the Treatment of Opioid Use Disorder: Maryland. http:// www.asam.org/docs/default-source/advocacy/State-medicaid-reports/Statemedicaid-reports_md.pdf. Accessed 01 March 2017.

21. American Society of Addiction Medicine, 2014g. Medicaid Coverage of Medications for the Treatment of Opioid Use Disorder: Massachusetts.
http://www.asam.org/docs/default-source/advocacy/State-medicaid-reports/ State-medicaid-reports_ma.pdf. Accessed 01 March 2017.

22. American Society of Addiction Medicine, 2014h. Medicaid Coverage of Medications for the Treatment of Opioid Use Disorder: Nebraska. http:// www.asam.org/docs/default-source/advocacy/State-medicaid-reports/Statemedicaid-reports_ne.pdf. Accessed 01 March 2017.

23. American Society of Addiction Medicine, 2014i. Medicaid Coverage of Mewdications for the Treatment of Opioid Use Disorder: Nevada. http:// www.asam.org/docs/default-source/advocacy/State-medicaid-reports/Statemedicaid-reports_nv.pdf. Accessed 01 March 2017.

24. American Society of Addiction Medicine, 2014j. Medicaid Coverage of Medications for the Treatment of Opioid Use Disorder: New York. http:/ www.asam.org/docs/default-source/advocacy/State-medicaid-reports/Statemedicaid-reports_ny.pdf. Accessed 01 March 2017.

25. American Society of Addiction Medicine, 2014k. Medicaid Coverage of Medications for the Treatment of Opioid Use Disorder: Tennessee. http:// www.asam.org/docs/default-source/advocacy/State-medicaid-reports/Statemedicaid-reports_tn.pdf. Accessed 01 March 2017.

26. American Society of Addiction Medicine, 2014l. Medicaid Coverage of Medications for the Treatment of Opioid Use Disorder: Vermont. http:// www.asam.org/docs/default-source/advocacy/State-medicaid-reports/Statemedicaid-reports_vt.pdf. Accessed 01 March 2017.

27. American Society of Addiction Medicine, 2014m. Medicaid Coverage of Medications for the Treatment of Opioid Use Disorder: Wisconsin. http:// www.asam.org/docs/default-source/advocacy/State-medicaid-reports/Statemedicaid-reports_wi.pdf. Accessed 01 March 2017.

28. WellCare of Florida, 2014. Florida Medicaid Comprehensive Preferred Drug List. https://florida.wellcare.com/WCAssets/florida/assets/fl_caid_pdl_ english_01_2014.pdf. Accessed 24 June 2017.

29. WellCare of Georgia, 2014. Georgia Medicaid Comprehensive Preferred Drug List. https://georgia.wellcare.com/WCAssets/georgia/assets/ga_caid_pdl_ english_01_2014.pdf. Accessed 24 June 2017.

30. Kaiser Family Foundation, n.d.-a. State Health Facts: Medicaid Benefits: Rehabilitation Services - Mental Health and Substance Abuse. http://www. kff.org/medicaid/state-indicator/rehabilitation-services-mental-health-andsubstance-abuse/. Accessed 24 June 2017.

31. American Hospital Association, n.d. AHA Hospital Statistics ${ }^{T M}$. http://www. ahadataviewer.com/book-cd-products/AHA-Statistics/. Accessed 24 June 2017.

32. Substance Abuse and Mental Health Services Administration, 2014. National Survey of Substance Abuse Treatment Services (N-SSATS): 2013. Data on substance abuse treatment Facilitities. BHSIS series S-73, HHS publication no. (SMA) 14-4890. https://www.samhsa.gov/data/sites/default/files/2013_NSSATS/2013_N-SSATS_National_Survey_of_Substance_Abuse_Treatment_ Services.pdf. Accessed 01 Sept 2017.

33. Substance Abuse and Mental Health Services Administration, 2015. National Survey of Substance Abuse Treatment Services (N-SSATS): 2014. Data on substance abuse treatment Facilitities. BHSIS series S-79, HHS publication no. (SMA) 16-4963. https://www.samhsa.gov/data/sites/default/files/2014_ National_Survey_of_Substance_Abuse_Treatment_Services/2014_National_ Survey_of_Substance_Abuse_Treatment_Services/2014_National_Survey_ of_Substance_Abuse_Treatment_Services.pdf. Accessed 01 Sept 2017.

34. Substance Abuse and Mental Health Services Administration, 2017. National Survey of Substance Abuse Treatment Services (N-SSATS): 2015. Data on substance abuse treatment Facilitities. BHSIS series S-88, HHS publication no. (SMA) 17-5031. https://wwwdasis.samhsa.gov/dasis2/nssats/2015_nssats_rpt. pdf. Accessed 01 Sept 2017.

35. Substance Abuse and Mental Health Services Administration, n.d. Number of DATA-Waived Practitioners Newly Certified Per Year. https://www.samhsa. gov/medication-assisted-treatment/physician-program-data/certifiedphysicians. Accessed 03 March 2017.

36. Kaiser Family Foundation, n.d-b. State Health Facts: Opioid Overdose Death Rates and All Drug Overdose Death Rates per 100,000 Population (AgeAdjusted). http://www.kff.org/other/state-indicator/opioid-overdose-deathrates. Accessed 02 November 2017.

37. Liebschutz JM, Crooks D, Herman D, Anderson B, Tsui J, Meshesha LZ, Dossabhoy S, Stein M. Buprenorphine treatment for hospitalized, opioiddependent patients. JAMA Intern Med. 2014;174:1369. https://doi.org/10. 1001/jamainternmed.2014.2556.

38. Mark TL, Dilonardo JD, Chalk M, Coffey RM. Factors associated with the receipt of treatment following detoxification. J Subst Abus Treat. 2003;24: 299-304. https://doi.org/10.1016/S0740-5472(03)00039-4. 
39. Agency for Healthcare Research and Quality, 2016c. Clinical Classifications Software (CCS) for ICD-9-CM. http://www.hcup-us.ahrq.gov/toolssoftware/ ccs/ccs.jsp. Accessed 26 April 2018.

40. Agency for Healthcare Research and Quality, 2016d. Elixhauser Comorbidity Software, Version 3.7. https://www.hcup-us.ahrq.gov/toolssoftware/ comorbidity/comorbidity.jsp. Accessed 26 April 2018.

41. Davis D, Carr D. State legal innovations to encourage naloxone dispensing J Amer Pharm Ass. 2017;57:S180-4. https://doi.org/10.1016/j.japh.2016.11.007.

42. Coffin PO, Sullivan SD. Cost-effectiveness of distributing naloxone to heroin users for lay overdose reversal. Ann Intern Med. 2013;158:1-9. https://doi. org/10.7326/0003-4819-158-1-201301010-00003.

43. Evans TI, Hadland SE, Clark MA, Green TC, Marshall BDL. Factors associated with knowledge of a good Samaritan law among young adults who use prescription opioids non-medically. Harm Reduct J. 2016;13(24). https://doi. org/10.1186/s12954-016-0113-2.

44. Cochran G, Gordon AJ, Lo-Ciganic W-H, Gellad WF, Frazier W, Lobo C, Chang $\mathrm{C}-\mathrm{CH}$, Zheng P, Donohue JM. An examination of claims-based predictors of overdose from a large Medicaid program. Med Care. 2017;55: 291-8. https://doi.org/10.1097/MLR.0000000000000676.

45. Dilokthornsakul P, Moore G, Campbell JD, Lodge R, Traugott C, Zerzan J, Allen R, Page RL. Risk factors of prescription opioid overdose among Colorado Medicaid beneficiaries. J Pain. 2016;17:436-43. https://doi.org/10. 1016/j.jpain.2015.12.006.

46. Lee S, Klein-Schwartz W, Welsh C, Doyon S. Medical outcomes associated with nonmedical use of methadone and buprenorphine. J Emerg Med. 2013:45:199-205. https://doi.org/10.1016/j.jemermed.2012.11.104.

47. Clark RE, Baxter JD, Aweh G, O'Connell E, Fisher WH, Barton BA. Risk factors for relapse and higher costs among Medicaid members with opioid dependence or abuse: opioid agonists, comorbidities, and treatment history. J Subst Abus Treat. 2015;57:75-80. https:/doi.org/10.1016/j.jsat.2015.05.001.

48. Knudsen HK, Abraham AJ, Oser CB. Barriers to the implementation of medication-assisted treatment for substance use disorders: the importance of funding policies and medical infrastructure. Eval Program Plann. 2011;34: 375-81. https://doi.org/10.1016/j.evalprogplan.2011.02.004.

49. Capoccia VA, Grazier KL, Toal C, Ford JH, Gustafson DH. Massachusetts's experience suggests coverage alone is insufficient to increase addiction disorders treatment. Health Aff. 2012;31:1000-8. https://doi.org/10.1377/ hlthaff.2011.0326.

50. Heslin KC, Owens PL, Karaca Z, Barrett ML, Moore B, Elixhauser A. Trends in opioid-related inpatient stays shifted after the U.S. transitioned to ICD-10CM diagnosis coding in 2015. Med Care. 2017;55:918-23. https://doi.org/10. 1097/MLR.0000000000000805.

Ready to submit your research? Choose BMC and benefit from:

- fast, convenient online submission

- thorough peer review by experienced researchers in your field

- rapid publication on acceptance

- support for research data, including large and complex data types

- gold Open Access which fosters wider collaboration and increased citations

- maximum visibility for your research: over $100 \mathrm{M}$ website views per year

At $\mathrm{BMC}$, research is always in progress.

Learn more biomedcentral.com/submissions 